\title{
EFFECT OF OVER-SAMPLING VERSUS UNDER-SAMPLING FOR SVM AND LDA CLASSIFIERS FOR ACTIVITY RECOGNITION
}

\author{
M.B ABIDINE ${ }^{1}$, B. FERGANI ${ }^{2}$ \& F.J ORDÓÑEZ 3 \\ ${ }^{1,2}$ Laboratoire d'Ingénierie des Systèmes Intelligents et Communicants, Faculty of Electronics and \\ Computer Sciences, USTHB, Algiers, Algeria. \\ ${ }^{3}$ Computer Science Department, University Carlos III of Madrid, Leganes, Madrid, Spain.
}

\begin{abstract}
Accurately recognizing the rare activities from sensor network-based smart homes for monitoring the elderly person is a challenging task. Activity recognition datasets are generally imbalanced, meaning certain activities occur more frequently than others. Not incorporating this class imbalance results in an evaluation that may lead to disastrous consequences for elderly persons. To overcome this problem, we evaluate two resampling methods using Over-sampling (OS) and Under-sampling (US). Then, these methods were combined with the discriminative classifiers named support vector machines (SVM) and linear discriminant analysis (LDA) experimental results carried out on multiple real-world smart home datasets demonstrate the feasibility of the proposal. Besides, a comparison with some state-of-the-art techniques based on Conditional Random Field (CRF) and Hidden Markov Model (HMM), we demonstrate that the US-SVM and OS-LDA are able to surpass HMM, CRF, SVM, LDA, OS-SVM and US-LDA. However, OS-LDA is the most effective method in terms of recognition of activities.

Keywords: humanactivity recognition, imbalanced data, LDA, machine learning, SVM.
\end{abstract}

\section{INTRODUCTION}

Recognition of activities of daily living (ADL) is one of the most important tasks in pervasive computing applications [1-4]. A research in human activity recognition, aimed to identify the physical activity such as cooking, brushing, dressing, bathing and so on, performed by human with real-time response.

Accurately recognizing the rare activities from sensor network-based smart homes for monitoring the elderly person is a challenging task. From the inference system perspective, one distinguishes two main streams of approaches for ADL recognition task in home setting. The first one advocates a knowledge-driven approach where the relationship between sensor features and activities follows a descriptive approach, for example, using ontology where activities are recognized through their linking to constraints on sensor events [5,6]. The second stream promotes a data-driven approach relying on machine learning and data mining techniques [7] to infer the underlying task where either supervised or unsupervised learning strategies are employed. In this way, as for any pattern recognition task, the keys to successful activity recognition are first to appropriately design a feature extraction strategy from the sensory data and, second, to design a suitable classifier that infers the user's activity and provides an interpretation of the observed sensor patterns. The latter commonly requires data labeling either for machine learning or evaluation purposes. For this purpose, annotation of data for classification task can be performed in many different ways, for example, use of cameras [8],

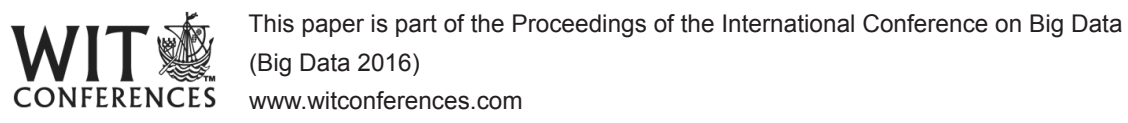

(C) 2016 WIT Press, www.witpress.com ISSN: 1755-7437 (paper format), ISSN: 1755-7445 (online), http://www.witpress.com/journals DOI: $10.2495 / \mathrm{DNE}-\mathrm{V} 11-\mathrm{N} 3-306-316$ 
self-reporting approaches, monitoring the diary activity [2], among others. Several classification algorithms have been employed for ADL recognition tasks [1-4], for example, Hiddenmarkov model (HMM) [2, 9], Conditional random fields (CRFs) [10], Linear discriminant analysis (LDA) [11], Sparse representation [12], Bayes approach [8], Neural network [13], Support vector machine [14] and its soft-margin multiclass SVM extension [15]. In ADL recognition task, the primary acknowledged limitation of classifier algorithms is the class imbalance problem [16]. This is due to the fact that sampling dataset is not evenly distributed among various classes, because of insufficient coverage of some classes, referred to as minority class (es) with respect to other majority (or dominant) class (es), which makes the classifier overwhelmed by the majority class (es) and ignores the minority class examples. For instance, in daily activity tasks, sleeping is generally done only once a day, while toileting is done several times a day. Consequently, any automated learning system may have difficulties in learning the concept related to the minority class (toileting). This trivially can result in a degradation of the performance of the automated activity classification algorithm (s).

In recent years, there have been many attempts to deal with the class imbalance problem [16-18]. Traditionally, research on this topic has mainly focused on a number of solutions both at the data and algorithmic levels. At the data level, solutions include many different forms of resampling such as OS (new samples are created for the minority class) and US (samples are eliminated for the majority class). At the algorithmic level, solutions include adjusting the costs associated with misclassification so as to improve performance [18].

Our objective is to avoid the overfitting caused by imbalanced class samples in order to perform automatic recognition of activities in a smart home. We evaluate two resampling methods at the data level: the OS and the US. These new schemes for activity recognition field are attractive as the only change is to the training data rather than to the algorithm itself. Then, these methods were combined with the Support Vector Machines (SVM) [19, 20] and LDA [21]. We used these discriminative methods for their simplicity-model and good performance with a fast prediction speed. HMM and CRF [2], are used as baseline methods. They have recently gained popularity in recognition activity field $[1,2]$.

Section 2 describes the notations used in this work, and the proposed approach using OS, US, SVM and LDA methods. Then, Section 3 presents the test setup and discusses the results acquired through a series of experiments using three different datasets. Finally, Section 4 concludes by summarizing our findings.

\section{DISCRIMINATIVEMETHODS FOR ACTIVITY RECOGNITION}

\subsection{Notation}

Our goal is to recognize ADL from sensor readings in a house [2]. The time space is discretized with intervals of a constant length $\mathrm{D} t$. We denote a sensor reading as $x_{t}^{i}$, indicating whether sensor $i$ fired at least once between time $t$ and time $t+\mathrm{D} t$, with $x_{t}^{i} \in\{0,1\}$. In a house with $n$ sensors installed, we define a binary vector $\vec{x}_{t}=\left(x_{t}^{1}, x_{t}^{2}, \ldots, x_{t}^{n}\right)^{T}$. An activity at time slice $t$ is denoted by $y_{t}$ with $y_{t} \in\{1, \ldots, N\}$ . The activity instances stored in the dataset have variable duration, every activity instance can be divided into a number of pieces, each of size $\mathrm{D} t$.

\subsection{Proposed approach for activity recognition}

Our approach combines the OS technique/US technique with the SVM/LDA classifier is shown in Fig.1. In the training phase, we perform the necessary pre-processing on the activity data represented 


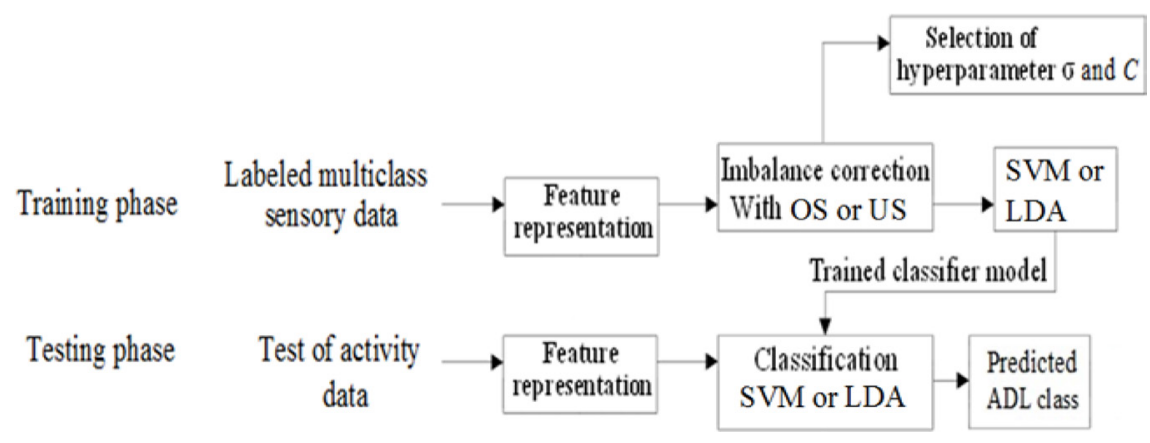

Figure 1: Diagram of our proposed approach.

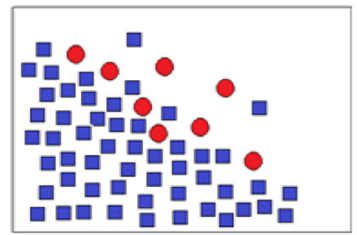

(a)

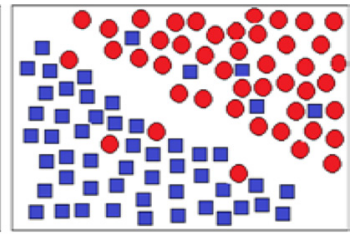

(b)

Figure 2: Over-sampling. (a) Before Over-sampling and (b) After Over-sampling.

in a feature space. On the pre-processed data, we need only to correct the class imbalance using the pre-classification named OS or US method. The balanced data is then used to learn the SVM classifier or used by the LDA classifier. It will then be used to process a new observation during the testing phase where the associated ADL class will be predicted.

\subsubsection{Handling the class imbalance problem}

Two data pre-processing non-heuristic methods can be used to balance in the number of samples in order to achieve abalanced training set: over sampling minority classes or under sampling majority classes.

2.2.1.1 Over-sampling: Oversampling approach increases the number of minority class samples. The simplest approach is random over-sampling, in which examples from the minority class are chosen randomly. Chosen examples are then duplicated from the minority class to the original set and added to the data training. All examples from the minority andmajority classes are kept, that means no information is lost, see figure 2.

2.2.1.2 Under-sampling (US): Under-sampling is an efficient method for class imbalance learning. This method uses a subset of the majority class to train the classifier. Since many majority class examples are ignored, the training set becomes more balanced and the training process becomes faster. The most common pre-processing technique is random majority under-sampling (RUS). In RUS, instances of the majority class are randomly discarded from the dataset, see figure 3. 


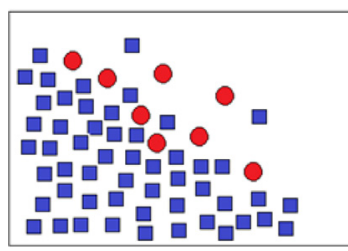

(a)

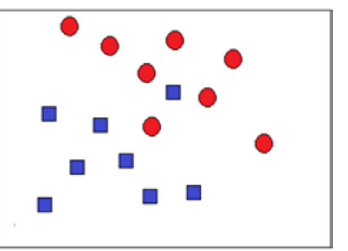

(b)

Figure 3: Under-sampling. (a) Before under-sampling and (b) after under-sampling.

\subsubsection{Classification}

2.2.2.1 Support vector machines For a two class problem (two types of activities $(N=2)$ ), we assume that we have a training set $\left\{\left(x_{i}, y_{i}\right)\right\}_{i=1}^{m}$ ( $m$ is the total number of data points), where $x \in R^{n}$ ( $n$ is the dimension of the input vectors) and $y_{i}$ are class labels either 1 or -1 . The primal formulation of SVM maximizes margin $2 / K(w, w)$ and minimizes the training error $\xi_{i}$ simultaneously by solving the following optimization problem

$$
\begin{aligned}
& \min _{w, b, \xi} 1 / 2 K(w, w)+C \sum_{i=1}^{m} \xi_{i} \\
& \text { subject to } y_{i}\left(w^{T} \varphi\left(x_{i}\right)+b\right) \geq 1-\xi_{i}, \xi_{i} \geq 0, i=1, \ldots, m
\end{aligned}
$$

The dual formulation of SVM can be reformulated as:

$$
\max _{\alpha_{i}} \quad \sum_{i=1}^{m} \alpha_{i}-\frac{1}{2} \sum_{i=1}^{m} \sum_{j=1}^{m} \alpha_{i} \alpha_{j} y_{i} y_{j} K\left(x_{i}, x_{j}\right)
$$

Subject to $\sum_{i=1}^{m} \alpha_{i} y_{i}=0$ and $0 \leq \alpha_{i} \leq C$, where $\alpha_{i}>0$ are Lagrange multipliers. The training samples for which Lagrangian multipliers are not zero are called support vectors. $\varphi($.$) is a non-$ linear function that coverts the sensor data in the lower dimensional space into a higher dimensional space.

The appropriate SVM classifier is selected by thekernel function $K(.,$.$) and the regularization$ parameters Cusing only training set, which maximizes the cross-validation rate in thespace of search. The kernel function can be of various types [20]. For the present study, the Gaussian kernel was selected due to its reported effectiveness in activity recognition [4]. We define the formulation of radial basis kernel function $(\mathrm{RBF})$ as follows: $K(x, y)=\exp \left(\frac{-1}{2 \sigma^{2}}\|x-y\|^{2}\right)$ where $\sigma$ is the width parameter. $C$ is regularization parameter. It is used to control the trade-off betw een margin and training error represented by slack variables $\xi_{i \text { in order to avoid }}$ the problem of overfitting.

Solving eqn (2) for $a$ gives a nonlinear decision function in the input spacefor classifying a test point $x \in R^{n}[20]$

$$
f(x)=\operatorname{sign}\left(\sum_{i=1}^{m_{\mathrm{sv}}} a_{i} y_{i} K\left(x, x_{i}\right)+b\right)
$$

Whose decision boundary is a hyperplane and translates to nonlinear boundaries in the original space and $m_{s v}$ is the number of support vectors $x_{i} \in R^{n}$. In this study, a software package LIBSVM [22] was used to implement the $N$ multiclass classifier algorithms. We used the one-versus-one 
method. This method consists in constructing $N(N-1) / 2$ classifiers and each one is trained on data from two classes. Then, a voting strategy is used for testing.

2.2.2.2 Linear discriminant analysis Given a set of observations in $n$-dimensional space: $D_{i}=\left\{x_{1}^{i}, \ldots, x_{m_{i}}^{i}\right\}\left(x_{j}^{i} \in R^{n}\right)$ from class $C_{i}(i=1, \ldots, N, N$ is the number of classes $)$, we assume that each of the class probability density functions can be modelled as normal distribution. Define the prior probabilities $p\left(C_{i}\right)$, means $\bar{m}_{i}$, and covariance matrices $\Sigma_{i}$ of each class:

$$
\begin{gathered}
p\left(\mathrm{C}_{i}\right)=\frac{m_{i}}{\sum_{j} m_{j}} \bar{m}_{i}=\frac{1}{m_{i}} \sum_{i=1}^{m_{i}} x_{i} \\
\Sigma_{i}=\frac{1}{m_{i}} \sum_{i=1}^{m_{i}}\left(x_{i}-\bar{m}\right)\left(x_{i}-\bar{m}\right)^{T}
\end{gathered}
$$

where $m_{i}$ is the number of patterns in class $C_{i}$. With LDA all classes are assumed to have the same covariance matrices $\Sigma_{i} \ldots, \Sigma_{N}$, on (5). We assign the new feature vector that is to be classified $x$ to $\mathrm{C}_{i}$ with the linear discriminant function $d_{i}$. This function is obtained by simplification the quadratic discriminant rule [9]

$$
d_{i}(x)=\log \left(p\left(\mathrm{C}_{i}\right)\right)-\frac{1}{2} m_{i}^{T} S_{W}^{-1} \bar{m}_{i}+x^{T} S_{W}^{-1} \bar{m}_{i}
$$

in which $\mathbf{S}_{w}$ is the common covariance matrix

$$
S_{W}=\sum_{i=1}^{N} \frac{m_{i}}{m-N} \Sigma_{i}
$$

The classification rule as given in eqn(8).

$$
f(x)=i^{*}: \Leftrightarrow i^{*}=\arg \max _{i} d_{i}(x)
$$

The classification rule for LDA method requires a training phase, meaning the computation of the discriminant functions and their parameters. The new data $x$ can be classified simply by solving the appropriate discriminant function for each class $\mathrm{C}_{i}$ and applying the classification rule (eqn(8)).

\section{EXPERIMENTAL RESULTS AND DISCUSSION}

In this section, we first give a description of the datasets and provide details of our experimental setup and then we present and discuss the results.

\subsection{Datasets}

For the experiments, we use an openly accessible datasets [23] gathered from a wireless sensor network with a single occupant. Data are collected using binary sensors such as reed switches to measure open-close states of doors and cupboards; pressure mats to measure sitting on a couch or lying in bed; float sensors to measure the toilet being flushed. Times at which no activity is annotated are referred to as 'Idle'. The data were collected by a Base-Station and labeled using a wireless Bluetooth headset 
combined with speech recognition software or handwritten activity diary. An overview of activities that were annotated, including the number of instances in the dataset, is shown in Table 1.

Table 1: Overview of activities and the number of observations for each dataset.

\begin{tabular}{|c|c|c|}
\hline TK26M (\%) & TK28M (\%) & TK57M (\%) \\
\hline Idle $_{(11.57)}$ & Idle $_{(23.65)}$ & Idle $_{(10.31)}$ \\
\hline Leaving $_{(56.53)}$ & Leaving $_{(45.77)}$ & Leaving $_{(45.27)}$ \\
\hline Toileting $_{(0.95)}$ & Toileting $_{(0.32)}$ & Eating $_{(1.42)}$ \\
\hline Showering $_{(0.66)}$ & Showering $_{(0.47)}$ & Toileting $_{(0.91)}$ \\
\hline Sleeping $_{(29)}$ & Brush teeth $_{(0.17)}$ & Showering $_{(0.72)}$ \\
\hline Breakfast $_{(0.27)}$ & Sleeping $_{(25.6)}$ & Brush \\
\hline $\operatorname{Dinner}_{(0.87)}$ & Dressing $_{(0.2)}$ & teeth $_{(0.38)}$ \\
\hline \multirow{11}{*}{$\operatorname{Drink}_{(0.15)}$} & Prep.Breakfast $_{(0.34)}$ & Shaving $_{(0.25)}$ \\
\hline & Prep.Dinner ${ }_{(0.38)}$ & Sleeping $_{(29.21)}$ \\
\hline & $\operatorname{Drink}_{(0.05)}$ & Dressing $_{(0.42)}$ \\
\hline & Dishes $_{(0.14)}$ & Medica- \\
\hline & Eat Dinner ${ }_{(0.22)}$ & $\operatorname{lin}_{(0.06)}$ \\
\hline & EatBreakfast $_{(06)}$ & Breakfast $_{(0.27)}$ \\
\hline & Play piano & $\operatorname{Lunch}_{(0.23)}$ \\
\hline & tay pranu $(2.07)$ & Dinner $_{(1.1)}$ \\
\hline & & Snack $_{(0.09)}$ \\
\hline & & $\operatorname{Drink}_{(0.13)}$ \\
\hline & & $\operatorname{Relax}_{(9.2)}$ \\
\hline
\end{tabular}

Table 2: Details of the houses in which the datasets.

\begin{tabular}{l|lll}
\hline Houses & TK26M & TK28M & TK57M \\
Age & 26 & 28 & 57 \\
Duration & 28 days & 13 days & 18 days \\
Sensors & 14 & 22 & 21 \\
Annotation & Bluetoothheadset & Bluetoothheadset & Handwrittendiary \\
\hline
\end{tabular}

\subsection{Setup and performance measures}

In these datasets, the sensor readings are divided in data segments of length $\mathrm{D} t=60 \mathrm{~s}$. This time slice duration is long enough to be discriminative and short enough to provide high accuracy labeling results. We separate the data into a test and training set using a 'leave one day out cross validation' strategy. In this strategy, one full day of sensor readings is used for testing and the remaining days $(l-1)$ are used for training; this is repeated $l$ times, with different training sets of size $(l-1)$ and report the average performance measure. 
Sensors outputs are binary and represented in a feature space which is used by the model to recognize the activities performed.We do not use the ' $R a w$ ' sensor data representation as observations; instead we use the concatenation of 'Change point' and 'Last' representations which have been shown to give much better results in activity recognition [2]. The raw sensor representation gives a 1 when the sensor is firing and a 0 otherwise. The 'change point' representation gives a 1 when the sensor reading changes. While the last sensor representation continues to assign a 1 to the last sensor that changed state until a new sensor changes state.

\subsection{Performance metrics}

As the activity instances were highly imbalanced between classes. We evaluate the performance of our models using the F-measure. We are dealing with a multi-classclassification problem and therefore define the notions of true positive (TP), false negatives (FN) and false positives (FP) for each class separately. With imbalanced data distribution, the overall accuracy metric at (9) is not relevant any more. These measures are calculated as follows:

$$
\begin{aligned}
\text { Accuracy } & =\frac{\sum_{i=1}^{N} T P_{i}}{\text { Total }} \times 100 \% \\
\text { Precision } & =\frac{1}{\mathrm{~N}} \sum_{i=1}^{N}\left[\frac{\mathrm{TP}_{i}}{\mathrm{TP}_{i}+\mathrm{FP}_{i}}\right] \times 100 \% \\
\text { Recall } & =\frac{1}{\mathrm{~N}} \sum_{i=1}^{N} \frac{\mathrm{TP}_{i}}{\mathrm{TP}_{i}+\mathrm{FN}_{i}} \times 100 \% \\
\mathrm{~F}-\text { Measure } & =\frac{2 . \text { Precision } \cdot \text { Recall }}{\text { Precision }+ \text { Recall }} \times 100 \%
\end{aligned}
$$

\subsection{Results}

We compared the performance of the HMM, CRF, SVM, OS-SVM, US-SVM, LDA, OS-LDA and US-LDA methods on the imbalanced datasets in which majority classes that occur less frequently typically have a longer duration and therefore take up more time(e.g. 'Idle', 'Leaving', 'Sleeping' and 'Relax'), while others are the minority classes.In our experiments, the SVM hyper-parameters $(\sigma, C)$ have been optimized in the range $(0.1-2)$ and $(0.1-1000)$. The parameters that yielded the highest class accuracy of leave-one-day-out cross validation technique were chosen during the training process. The best pair parameters $\left(\sigma_{o p t}, C_{o p t}\right)=(1.7,5),(1,10)$ and $(2,1)$ are used for the datasets TK26M, TK28M and TK57M, respectively. The overall performance of our approach on different datasets in terms of Recall, Precision, F-measure, and accuracy obtained with the concatenation of the 'Change point+Last' representation during the testing phase is summarized in Table 3.

This table shows that, both the US-SVM and OS-LDA methods perform better in terms of F-measure, while SVM perform better in terms of accuracy for all datasets because this method is affected by the majority activities. 
Table3: Accuracy (Acc.), Recall, Precision (Prec.) and F-measure (F-Meas.) results for all classifiers. Bold values are the best values for different criteria.

\begin{tabular}{llcccc}
\hline Datasets & Methods & Acc $(\%)$ & Recall (\%) & Prec & F-Meas \\
\hline \multirow{7}{*}{ TK26M } & HMM [24] & 92 & 66 & 68 & 67 \\
& CRF [24] & 91 & 57 & 68 & 62 \\
& SVM & $\mathbf{9 4 . 8}$ & 61.8 & 65.9 & 63.8 \\
& OS-SVM & 91.4 & 72.3 & 73.9 & 73.1 \\
& US-SVM & 91.0 & 74.4 & 77.2 & 75.8 \\
& LDA & 92.4 & 72.3 & 73.7 & 73.0 \\
& OS-LDA & 91.3 & $\mathbf{7 5 . 0}$ & $\mathbf{7 8 . 4}$ & $\mathbf{7 6 . 7}$ \\
& US-LDA & 56.2 & 46.8 & 52.3 & 49.4 \\
\hline \multirow{7}{*}{ TK28M } & HMM [24] & 80 & $\mathbf{5 5}$ & 42 & 48 \\
& CRF [24] & 92 & 46 & 54 & 49 \\
& SVM & $\mathbf{8 5 . 5}$ & 39.3 & 37.5 & 38.4 \\
& OS-SVM & 63.1 & 46.7 & 48.3 & 47.5 \\
& US-SVM & 59.1 & 48.5 & 50.3 & 49.4 \\
& LDA & 80.6 & 48.7 & 49.5 & 49.1 \\
& OS-LDA & 55.5 & 50.2 & $\mathbf{5 5 . 4}$ & $\mathbf{5 2 . 7}$ \\
& US-LDA & 08.6 & 16.8 & 46.4 & 24.7 \\
\hline & HMM [24] & 76 & 40 & 37 & 39 \\
& CRF [24] & 78 & 30 & 36 & 33 \\
& SVM & $\mathbf{8 0 . 8}$ & 35.6 & 34.9 & 35.2 \\
OS-SVM & 77.0 & 37.4 & 34.1 & 35.7 \\
TK57M & US-SVM & 76.3 & 40.8 & 33.8 & 37.0 \\
& LDA & 79.1 & 40.7 & 37.2 & 38.9 \\
& OS-LDA & 76.4 & $\mathbf{4 1 . 5}$ & $\mathbf{4 4 . 8}$ & $\mathbf{4 3 . 1}$ \\
& US-LDA & 34.5 & 16.7 & 27.2 & 20.7 \\
\hline & & & & & \\
\hline
\end{tabular}

In order to find out which activities are relatively harder to be recognized, we report in Figs 4 and 5 the classification results in terms of accuracy measure for each activity with SVM, US-SVM, LDA and OS-LDA methods for TK26M and TK57M datasets. In Fig. 4, for the US-SVM, the minority activities 'Toileting', 'Showering' and the kitchen activities 'Breakfast', 'Dinner', 'Drink', are significantly better detected comparatively with SVM. OS-LDA gave better results for the kitchen activities. The majority activities are better for all methods while the 'Idle' activity is more accurate for SVM compared to other methods.We can see in Fig.5 that the minority activities ('Eating', 'Showering', 'Shaving', 'Medication' and the kitchen activities ('Breakfast', 'Lunch', 'Snack' and 'Drink')) are better recognized with US-SVM comparatively to SVM method. OS-LDA has improved the classification of minority activities ('Brush teeth', 'Dressing', all kitchen activities, 'Relax') compared to LDA method. 
It can beseen that the 'Medication', 'Snack' and 'Drink' activities with 16, 24 and 34 instances, respectively, have not been recognized at all with the SVM method. Finally, we note that most confusion obtained with these recognition methods takes place in the kitchen activities. They perform worst and are in general hard to recognize.

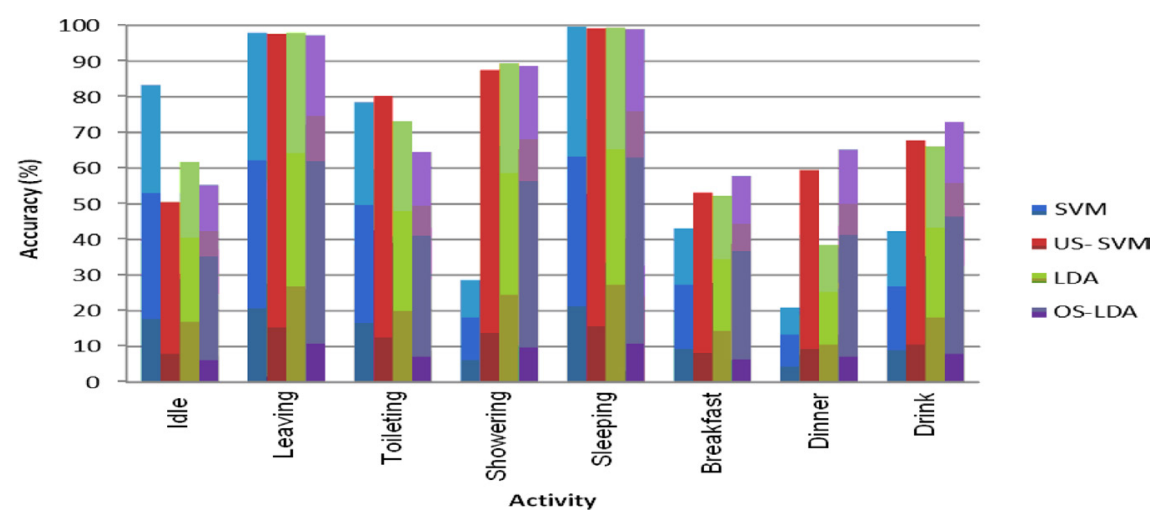

Figure 4: Comparison of accuracy measure for each activity between SVM, US-SVM, LDA and OS-LDA with TK26M dataset.

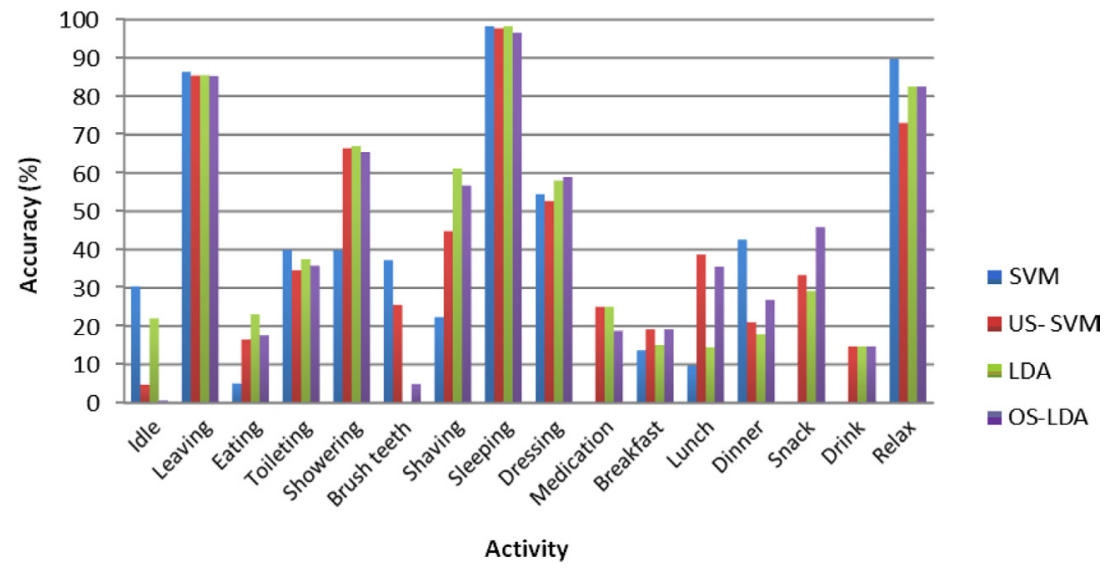

Figure 5: Comparison of accuracy measure for each activity between SVM, US-SVM, LDA and OS-LDA with TK57M dataset.

\subsection{Discussion}

In this section, we explain the difference in terms of performance between different recognition methods. Our results give us early experimental evidence that LDA outperforms SVM for classifying the activities. SVM is more sensitive than the LDA on imbalanced data because the constraint in (eqn(1)) imposes equal total influence (i.e. $C$ ) from the positive and negative support vectors. This would cause the separating hyperplane to be skewed toward the minority class. This affects the SVM classifier performances and favorites the classification of majority activities ('Idle', 'Leaving', 'Sleeping' and 'Relax'). 
The resampling methods : OS and US overcome the class imbalance problem for SVM and LDA classifiers for all databases with OS-LDA is slightly higher than US-SVM in terms of activity recognition. It has been shown that US-SVM outperforms US-LDA because SVM fits better on the balanced training set with little data as opposed to LDA perform worst. US method has a negative effect on the classification performance of LDA. The reason is due to the low number of samples is not sufficient for estimating the parameters of normal distribution across multiple classes. OS-LDA outperforms OS-SVM because SVM method is sensitive to overfitting due to duplication samples of the training set with the OS algorithm. Therefore, the over-sampling is more suited to LDA classification method.

The recognition of the kitchen activities as 'Breakfast' 'Dinner' and 'Drink' is lower compared to the others activities for all methods. The kitchen activities are food-related tasks, they are worst recognized because most of the instances of these activities were performed in the same location (kitchen) using the same set of sensors. For example, 'Toileting' and 'Showering' are more separable because they are in two different rooms, which make the information from the door sensors enough to separate the two activities. Therefore the location of sensors strongly influences recognition performance.

\section{CONCLUSIONS}

Our experiments showed very impressive results using OS-LDA and US-SVM pattern recognition methods to improve prediction accuracy for imbalanced human activity datasets. Also, we showed that the OS-LDA is better than the other classifiers to classify multiclass sensory data. It is found that SVM is more sensitive to overfitting on a dominant class. We observed also that differences in the layout of houses can greatly affect the performance in activity recognition. Finally, it would be valuable to compare this approach with the combination OS/US with HMM/CRF classification methods.

\section{REFERENCES}

[1] Tapia, E.,Intille, S. \& Larson, K., Activity recognition in the home using simple and ubiquitous sensors. In Pervasive Computing Lecture Notes in Computer Science, Springer Heidelberg, 3001, pp. 158-175, 2004. http://dx.doi.org/10.1007/978-3-540-24646-6_10

[2] van Kasteren, T., Noulas, A., Englebienne, G. \& Krose, B., Accurate activity recognition in a home setting. In UbiComp, 08, New York, NY, USA: ACM, pp. 1-9, 2008. http://dx.doi.org/10.1145/1409635.1409637

[3] Abidine, M.B., Fergani, L., Fergani, B. \& Fleury, A., Improving human activity recognition in smart homes. International Journal of E-Health and Medical Communications (IJEHMC), 6(3), pp. 19-37, 2015. http://dx.doi.org/10.4018/IJEHMC.2015070102

[4] Fleury, A., Vacher, M. \& Noury, N., SVM-based multi-modal classification of activities of daily living in health smart homes: sensors, algorithms and first experimental results. IEEE Transactions on Information Technology in Biomedicine, 14(2), pp. 274-283, 2010. http://dx.doi.org/10.1109/TITB.2009.2037317

[5] Aggrawal, J.K. \& Ryoo, M.S., Human activity analysis: a review. ACM Computing Surveys, 43(3), pp. 1-16, 2011. http://dx.doi.org/10.1145/1922649.1922653

[6] Chen, L., Nugent, C.D. \& Wang, H., A knowledge-driven approach to activity recognition in smart homes. IEEE Transactions on Knowledge and Data Engineering, 24, pp. 961-974, 2012. http://dx.doi.org/10.1109/TKDE.2011.51 
[7] Bishop, C., Pattern Recognition and Machine Learning, Springer: New York, ISBN: 978-0387-31073-2, 2006.

[8] Logan, B., Healey, J., Philipose, M., Tapia, E.M. \& Intille, S.S., A long-term evaluation of sensing modalities for activity recognition In Ubicomp '07, pp. 483-500, 2007.

[9] Huang, W., Zhang, J. \& Liu, Z., Activity recognition based on hidden Markov models. In Knowledge Science, Engineering and Management, Springer Berlin Heidelberg, pp. 532-537, 2007. http://dx.doi.org/10.1007/978-3-540-76719-0_54

[10] Vail, D.L., Veloso, M.M. \& Lafferty, J.D., Conditional random fields for activity recognition. In International Conference on Autonomous Agents and Multi-agent Systems (AAMAS), 2007. http://dx.doi.org/10.1145/1329125.1329409

[11] Abidine, M.B. \& Fergani, B., Evaluating C-SVM, CRF and LDA classification for daily activity recognition. In Proceeding of IEEE ICMCS, Tangier-Morocco, pp. 272-277, 2012.

[12] Liu, S., Gao, R.X., John, D., Staudenmayer, J. \& Freedson, P.S., Classification of physical activities based on sparse representation. In 2012 Annual International Conference of the IEEE Engineering in Medicine and Biology Society (EMBC), pp. 6200-6203, 2012.

[13] Fang, H. \& He, L., BP neural network for human activity recognition in smart home. In 2012 International Conference on Computer Science \& Service System (CSSS), pp. 1034-1037, 2012. http://dx.doi.org/10.1109/CSSS.2012.262

[14] Fleury, A., Vacher, M. \& Noury, N., SVM-based multi-modal classification of activities of daily living in health smart homes: sensors, algorithms and first experimental results. IEEE Transactions on Information Technology in Biomedicine, 14(2), pp. 274-283, 2010. http://dx.doi.org/10.1109/TITB.2009.2037317

[15] Abidine, M.B., Fergani, B. \& Clavier, L., Importance-Weighted the imbalanced data for $c$-svm classifier to human activity recognition. Proceeding of IEEE WOSSPA'13, pp. 330-335, 2013. http://dx.doi.org/10.1109/wosspa.2013.6602386

[16] Chawla, N.V., Data mining for imbalanced datasets: an overview. In Data Mining and Knowledge Discovery Handbook, Springer, pp. 875-886, 2010.

[17] Akbani, R., Kwek, S. \& Japkowicz, N., Applying support vector machines to imbalanced datasets. In Machine Learning: ECML, Springer Berlin Heidelberg, pp. 39-50, 2004. http://dx.doi.org/10.1007/978-3-540-30115-8_7

[18] Huang, Y.M. \& Du, S.X., Weighted support vector machine for classification with uneven training class sizes. In Proceedings of the IEEE ICMLC, 7, pp. 4365-4369, 2005.

[19] Bishop, C., Pattern Recognition and Machine Learning, Springer: New York, ISBN: 978-0387-31073-2, 2006.

[20] Vapnik, V.N., The Nature of Statistical Learning Theory, (Statistics for Engineering and Information Science), Springer Verlag, 2000. http://dx.doi.org/10.1007/978-1-4757-3264-1

[21] Hastie, T., Tibshirani, R. \& Friedman, J.H., The Elements of Statistical Learning, Springer, Heidelberg: New York, 2001. http://dx.doi.org/10.1007/978-0-387-21606-5

[22] Chang, C.C. \& Lin, C.J., LIBSVM, available at http://www.csie.ntu.edu.tw/ cjlin-/libsvm/

[23] Datasets for Activity Recognition, available at http://sites.google.com/site/tim0306/ (accessed February 09, 2012)

[24] Van Kasteren, T., Alemdar, H. \& Ersoy, C., Effective performance metrics for evaluating activity recognition methods. In Proceedings of the 24th ARCS 2011, Comot, Italy, p. 10, 2011. 